\title{
On the Forced Response of Harmonically and Partially Mistuned Bladed Disks. Part II: Partial Mistuning and Applications*
}

\author{
MARC P. MIGNOLET ${ }^{\mathrm{a}, \dagger}$, WEI HU $^{\mathrm{a}}$ and IOAN JADIC ${ }^{\mathrm{b}}$ \\ a Department of Mechanical and Aerospace Engineering, Arizona State University, Tempe, Arizona 85287-6106, USA; \\ ${ }^{\mathrm{b}}$ Raytheon Aircraft Company, P.O. Box 85, Wichita, Kansas 67201-0085, USA
}

(Received in final form 17 July 1998)

\begin{abstract}
This series of two papers focuses on the determination and clarification of the characteristics of the forced response of bladed disks that exhibit a mistuning pattern that is either harmonic or partial. Harmonic mistuning refers to single wavelength variations in structural properties along the disk while partial mistuning is associated with blade characteristics that are random in a specific sector and tuned elsewhere. The results of this analysis demonstrate that many features of the response of these simple systems match not only qualitatively but also quantitatively their counterparts on randomly mistuned bladed disks. Relying on these similarities, simple and reliable approximations of the localization factor and of the mean response are easily derived that exemplify the usefulness of harmonic and partial mistuning patterns. Finally, it is demonstrated both theoretically and by comparison with simulation results that the maximum amplitude of response of a disk closely follows a Weibull-type distribution in all coupling situations, from very weak to very strong.
\end{abstract}

Keywords: Bladed disks, Blade vibration, Mistuning, Localization phenomenon, Maximum response

\section{PARTIAL MISTUNING}

The localization of both the free and forced responses of weakly coupled mistuned bladed disks which was exemplified in the companion paper, Figs. I.2-I.7, is symptomatic of a general lack of transmission of "information" across the disk, especially when the damping effects dominate. This property implies in particular that the response of a specific blade will only depend on the properties of a few of its neighbors. It is thus conjectured that the behavior of the $j$ th blade of a weakly coupled disk can be closely represented by its response for the partially mistuned disk with the given deviations in

\footnotetext{
* This paper was originally presented at ISROMAC-7.

${ }^{\dagger}$ Corresponding author. Tel.: (480) 965-1484. Fax: (480) 965-1384. E-mail: mignolet@enuxsa.eas.asu.edu.

${ }^{1}$ To avoid duplication, all equations and figures marked with a roman numeral $I$ in this part will refer to the equations and figures with the same numbers in Part I.
} 
structural properties $\delta k_{l}, l=j, j \pm 1, \ldots, j \pm M$, and $\delta k_{l}=0, l \neq j, j \pm 1, \ldots, j \pm M$.

To demonstrate the validity of this assumption, simulation studies were conducted on the system shown in Fig. I.1 with different values of the coupling stiffness. Displayed in Figs. 1 and 2 are the probability density functions of the amplitude of blade response obtained by Monte Carlo simulations of the fully and 3-blade $(M=1)$ mistuned disks with the values $\tilde{F}_{0}=1 \mathrm{~N}$ and $r=3$ which will be used throughout this second part. Clearly, for very low coupling stiffnesses $\left(k_{\mathrm{C}}=2,000 \mathrm{~N} / \mathrm{m}\right.$ in Fig. 1), the agreement is excellent over the entire range of amplitudes. As the level of coupling is increased, see Fig. 2 for $k_{\mathrm{C}}=4,543 \mathrm{~N} / \mathrm{m}$, the accuracy of this approximation deteriorates, especially near the peak of the distribution, but it still provides a reasonable model of the exact probability density function. Extending the size of the mistuning zone to five blades $(M=2)$ provides an improved approximation of the distribution of the response, especially near the peak, as can be seen in Fig. 2.

The very reliable results shown in Figs. 1 and 2 motivate an analysis of the computational aspects of the determination of the response of partially mistuned bladed disks. For such systems, the equation of motion, Eq. (I.6), can be written in the form

$$
\begin{aligned}
& -k_{\mathrm{C}} \mathrm{e}^{\mathrm{i} \Delta \psi} \tilde{X}_{n+1}-k_{\mathrm{C}} \mathrm{e}^{-\mathrm{i} \Delta \psi} \tilde{X}_{n-1} \\
& +\left(2 k_{\mathrm{C}} \cos \Delta \psi+\mathrm{i} \omega c\right) \tilde{X}_{n}=\tilde{F}_{0}-\sum_{l=j-M}^{j+M}\left(\delta k_{l} \tilde{X}_{l}\right) \delta_{n l}
\end{aligned}
$$

for $n=1,2, \ldots, N$. In this representation, each of the mistuning terms $\delta k_{l} \tilde{X}_{l}$ appears as a force, of unknown magnitude, acting only on the corresponding blade of the tuned disk. This interpretation of the equation of motion of the system leads to

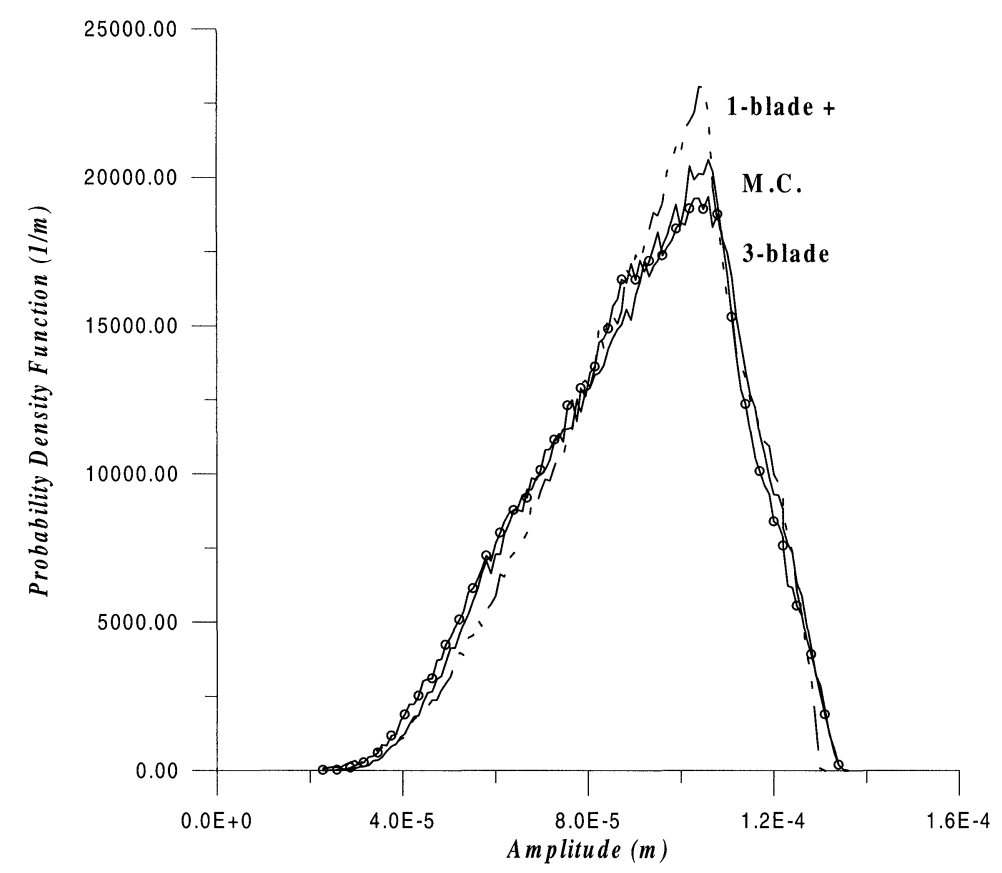

FIGURE 1 Probability density function of the amplitude of response obtained by Monte Carlo simulation of fully random disk ("M.C."), with 3-blade mistuning ("3-blade"), and with modified 1-blade mistuning (“1-blade + ") $k_{\mathrm{C}}=2,000 \mathrm{~N} / \mathrm{m} ; \sigma_{\delta k}=8,000 \mathrm{~N} / \mathrm{m}$, $c=1.443 \mathrm{Ns} / \mathrm{m}$ 


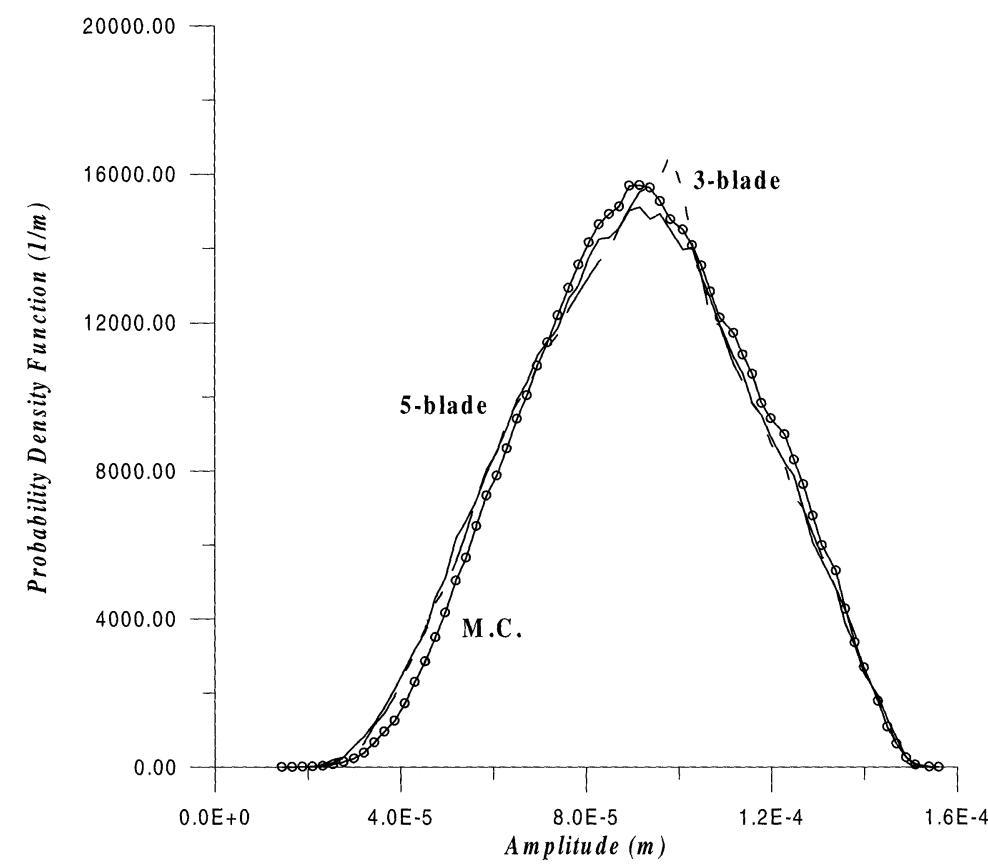

FIGURE 2 Probability density function of the amplitude of response obtained by Monte Carlo simulation of fully random disk ("M.C."), with 3-blade mistuning ("3-blade"), and with 5-blade mistuning ("5-blade”) $k_{\mathrm{C}}=4,543 \mathrm{~N} / \mathrm{m} ; \sigma_{\delta k}=8,000 \mathrm{~N} / \mathrm{m}$, $c=1.443 \mathrm{Ns} / \mathrm{m}$.

an implicit expression for its response as

$$
\tilde{X}_{n}=\tilde{X}_{n \mathrm{t}}-\sum_{l=j-M}^{j+M}\left(\delta k_{l} \tilde{X}_{l}\right) \tilde{h}_{n-l} \quad n=1,2, \ldots, N,
$$

where $\tilde{X}_{n \mathrm{t}}$ is the tuned response of blade $n$, i.e.

$$
\tilde{X}_{n \mathrm{t}}=\frac{\tilde{F}_{0}}{\mathrm{i} c \omega} .
$$

The above equations provide an efficient computational tool for the prediction of the response of partially mistuned disks. Indeed, expressing Eq. (2) for $n=j, j \pm 1, \ldots, j \pm M$ yields the relations

$$
\begin{gathered}
\sum_{l=j-M}^{j+M}\left(\delta k_{l} \tilde{h}_{n-l}+\delta_{n l}\right) \tilde{X}_{l}=\frac{\tilde{F}_{0}}{\mathrm{i} c \omega} \\
n=j, j \pm 1, \ldots, j \pm M,
\end{gathered}
$$

which constitute a set of $(2 M+1)$ linear complex algebraic equations in the $(2 M+1)$ unknowns $\tilde{X}_{n}$, $n=j, j \pm 1, \ldots, j \pm M$. Once these values have been determined, the response of the remaining blades can be obtained directly from Eq. (2). Note that this procedure is computationally very efficient since it involves only a $(2 M+1) \times(2 M+1)$ linear system of equations, as opposed to an $N \times N$ one in the fully mistuned situation.

The numerical results obtained with 3-blade mistuning and presented in Figs. 1 and 2 motivate the analytical investigation of an even simpler approximation, i.e. the single-blade mistuning model $(M=0)$. From Eq. (4), it is directly found that the response of the mistuned blade is

$$
\tilde{X}_{j}=\frac{\tilde{F}_{0}}{\mathrm{i} c \omega} \frac{1}{1+\delta k_{j} \tilde{h}_{0}} .
$$

Then, introducing this expression in Eq. (2) yields the response of the tuned blade $l \neq j$ as

$$
\tilde{X}_{l}=\frac{\tilde{F}_{0}}{\mathrm{i} c \omega}\left[1-\frac{\delta k_{j} \tilde{h}_{l-j}}{1+\delta k_{j} \tilde{h}_{0}}\right] \quad l \neq j,
$$


where the impulse response values $\tilde{h}_{l}$ are given by Eq. (I.12). A comparison of the probability density functions of the amplitude of the blade $j$ corresponding to a fully mistuned bladed disk and to Eq. (5), not presented here for brevity, has shown that the 1-blade mistuning model is oversimplified: Indeed, it fails to account properly, even at very low coupling levels (e.g. $k_{\mathrm{C}}=454 \mathrm{~N} / \mathrm{m}$ ), for the increase in blade response that represents the most important aspect of mistuning. The combination of these poor results and the good ones shown in Figs. 1 and 2 demonstrates that the accurate prediction of the effects of mistuning requires the consideration of at least 3 consecutive blades with dissimilar properties.

The great simplicity of the analytical results presented in Eqs. (5) and (6) has motivated the formulation of a simplified 3-blade mistuned model in which the response of the middle one is expressed as the sum of its 1-blade approximation, Eq. (5), and of the increment in response from the tuned value generated by the fluctuations in structural properties of the two neighborhooding blades taken one at a time. That is

$$
\begin{aligned}
\tilde{X}_{j}= & \left(\tilde{X}_{j}\right)_{\delta k_{j} \neq 0}+\left[\left(\tilde{X}_{j}\right)_{\delta k_{j+1} \neq 0}-\tilde{X}_{j \mathrm{t}}\right] \\
& +\left[\left(\tilde{X}_{j}\right)_{\delta k_{j-1} \neq 0}-\tilde{X}_{j \mathrm{t}}\right] .
\end{aligned}
$$

Using Eqs. (5) and (6), this approximation reduces to

$$
\begin{gathered}
\tilde{X}_{j}=\frac{\tilde{F}_{0}}{\mathrm{i} c \omega}\left[\frac{1}{1+\delta k_{j} \tilde{h}_{0}}-\frac{\delta k_{j+1} \tilde{h}_{N-1}}{1+\delta k_{j+1} \tilde{h}_{0}}\right. \\
\left.-\frac{\delta k_{j-1} \tilde{h}_{1}}{1+\delta k_{j-1} \tilde{h}_{0}}\right] .
\end{gathered}
$$

The probability density functions of the amplitude of response determined from this model for $k_{\mathrm{C}}=2,000 \mathrm{~N} / \mathrm{m}$ and $k_{\mathrm{C}}=454 \mathrm{~N} / \mathrm{m}$ are plotted in Figs. 1 and 3 together with the Monte Carlo simulation results for the fully mistuned disks. From these

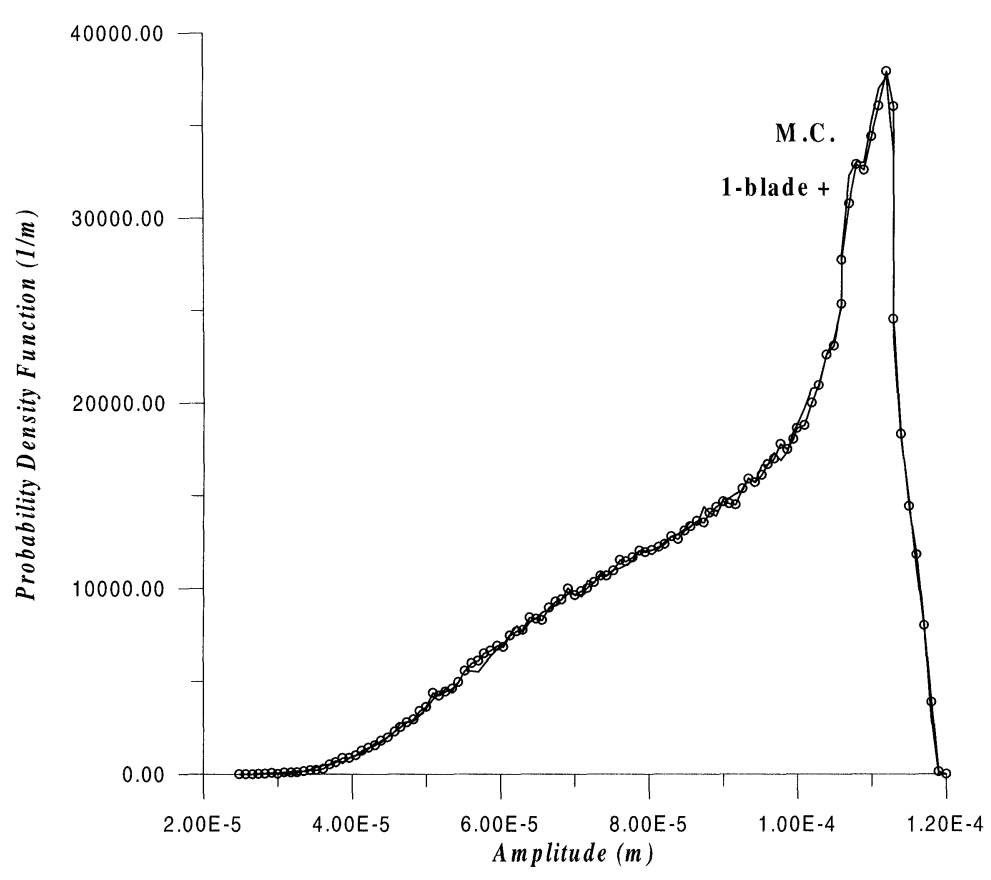

FIGURE 3 Probability density function of the amplitude of response obtained by Monte Carlo simulation of fully random disk ("M.C.") and with modified 1-blade mistuning ("1-blade +") $k_{\mathrm{C}}=454 \mathrm{~N} / \mathrm{m} ; \sigma_{\delta k}=8,000 \mathrm{~N} / \mathrm{m}, c=1.443 \mathrm{Ns} / \mathrm{m}$. 


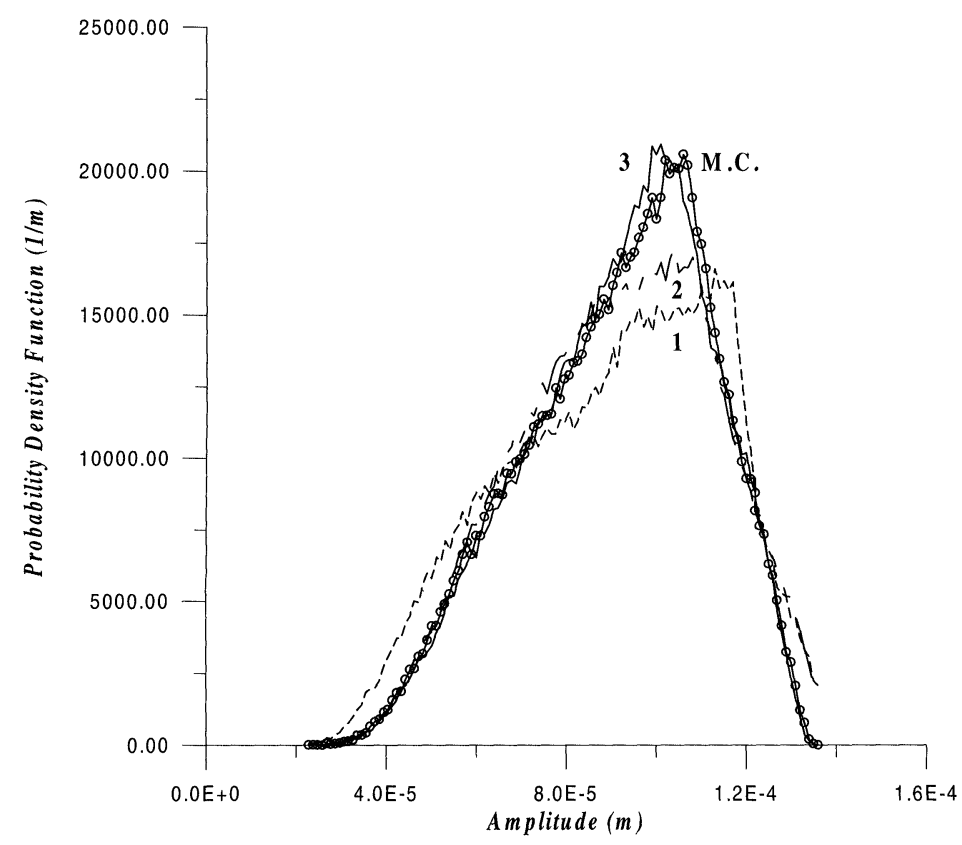

FIGURE 4 Probability density function of the amplitude of response obtained by Monte Carlo simulation of fully random disk ("M.C.") and from perturbation analyses accurate to order $k_{\mathrm{C}}^{1}, k_{\mathrm{C}}^{2}$, and $k_{\mathrm{C}}^{3} ; k_{\mathrm{C}}=2,000 \mathrm{~N} / \mathrm{m} ; \sigma_{\delta k}=8,000 \mathrm{~N} / \mathrm{m}, c=1.443 \mathrm{Ns} / \mathrm{m}$.

plots, it is concluded that the simple representation of the forced response given by Eq. (8) provides a reliable basis for the assessment of the effects of mistuning in weakly coupled systems. Further, as expected, the accuracy of this approximation improves as the coupling stiffness is reduced and is excellent for very weakly coupled systems, see Fig. 3 .

The various numerical results presented in this section have clearly demonstrated the validity of the basic assumption underlying the present analysis, i.e. the limited range of influence of the mistuning of a specific blade in weakly coupled disks. A similar observation could be drawn from available perturbation results (Wei and Pierre, 1988; Castanier and Pierre, 1993) which indicate that the approximation of order $k_{\mathrm{C}}^{\eta}$, i.e. with an error of order $O\left(k_{\mathrm{C}}^{\eta+1}\right)$, of the forced response of blade $j$ involves the mistuning terms $\delta k_{l}, l \in[j-\eta$, $j+\eta]$. In this light, it could be misconstrued that the exact analysis of a partially, $2 M+1$-blade, mistuned bladed disk yields results similar to a perturbation analysis correct to order $k_{\mathrm{C}}^{M}$ of the fully mistuned system. In fact, it can be seen from
Figs. 1 and 4 that the 3-blade mistuning model yields an approximation of the exact, full disk, probability density function that is much better than the order $k_{\mathrm{C}}$ perturbation expression, it actually appears equivalent to its $k_{C}^{3}$ counterpart!

\section{APPLICATIONS}

\section{Computation of the Localization Factor, $\delta>\sigma$}

The degree of localization in a nearly periodic assembly can be quantified by the localization factor $\gamma$ defined as

$$
\gamma=-\frac{1}{n} \ln \left|\frac{\tilde{h}_{n}^{\prime}}{\tilde{F}_{00}}\right|,
$$

where $\tilde{h}_{n}^{\prime}$ is the amplitude of the steady state response of the $n$th assembly corresponding to a harmonic excitation of magnitude $\tilde{F}_{00}$ applied on the first one. The determination of $\gamma$ in a randomly perturbed system is in general intractable but asymptotic results can be derived in the limits of 
weak and strong coupling. In the case of a chain of damped single-degree-of-freedom systems, it has been shown by Castanier and Pierre (1993) that

$$
\begin{aligned}
& \gamma \approx \frac{1}{\sqrt{\alpha(4-\alpha)}}\left(\frac{\delta}{R}\right)+\frac{1}{2 \alpha(4-\alpha)}\left(\frac{\sigma}{R}\right)^{2} \\
& \quad \text { for } \frac{\sigma}{R} \ll 1 ; \frac{\delta}{R} \ll 1
\end{aligned}
$$

and

$$
\begin{aligned}
\gamma= & -1+\frac{1}{4}\left[\frac{2-\alpha}{\sqrt{3}(\sigma / R)}+1\right] \\
& \times \ln \left[\left(2-\alpha+\sqrt{3}\left(\frac{\sigma}{R}\right)\right)^{2}+\left(\frac{\delta}{R}\right)^{2}\right] \\
- & \frac{(\delta / R)}{2 \sqrt{3}(\sigma / R)} \arctan \left[\frac{2-\alpha-\sqrt{3}(\sigma / R)}{(\delta / R)}\right] \\
- & \frac{1}{4}\left[\frac{2-\alpha}{\sqrt{3}(\sigma / R)}-1\right] \ln \left[\left(2-\alpha-\sqrt{3}\left(\frac{\sigma}{R}\right)\right)^{2}\right. \\
& \left.+\left(\frac{\delta}{R}\right)^{2}\right]+\frac{(\delta / R)}{2 \sqrt{3}(\sigma / R)} \\
& \times \arctan \left[\frac{2-\alpha+\sqrt{3}(\sigma / R)}{(\delta / R)}\right]
\end{aligned}
$$

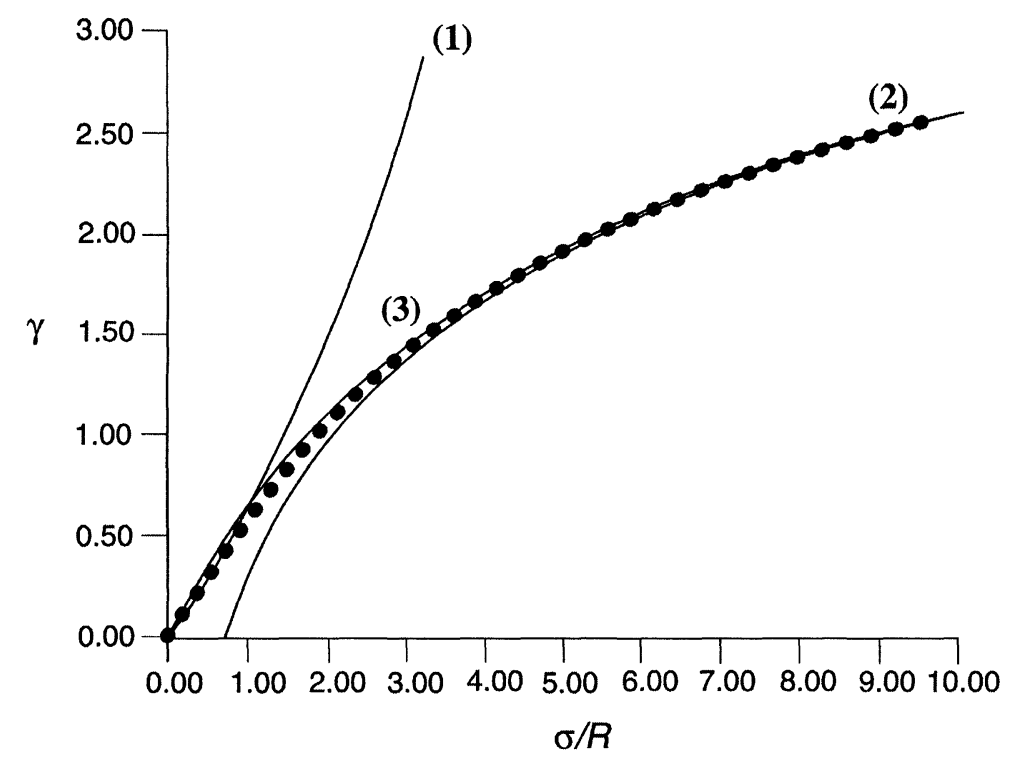

FIGURE 5 Localization factor as a function of $\sigma / R=\delta / R$ corresponding to the weak ("1") and strong ("2") localization limits, to the alternate mistuning model ("3"), and to Monte Carlo simulation ("•", from Castanier and Pierre, 1993). for $\sigma / R \gg 1 ; \delta / R \gg 1$ when

$$
\alpha=\frac{m \omega^{2}-k_{\mathrm{t}}}{k_{\mathrm{C}}} \in[0,4] .
$$

These two expressions and the exact values of the localization factor computed by Monte Carlo simulation by Castanier and Pierre (1993) are shown in Fig. 5 as a function of $\sigma / R$ when $\sigma / R=$ $\delta / R$. Clearly, Eqs. (10)-(12) provide accurate approximations of the localization factor in the limits $\sigma / R=\delta / R \ll 1$ and $\sigma / R=\delta / R \gg 1$ but cannot reliably be used in the domain $\sigma / R=\delta / R \in[1,3]$. The understanding and prediction of the effects of mistuning in this transition region are very important issues since the maximum amplification of the forced response has been observed to occur for such values of the system parameters.

It was demonstrated earlier (see Figs. I.2-I.7) that the response of mistuned bladed disks is dictated by the very short wavelength fluctuations in the stiffnesses when the damping effects are dominant in the localization of the system, i.e. when $\delta \geq \sigma$. This observation suggests that the physical characteristics, such as localization factor, 
of alternatively mistuned, weakly coupled bladed disks should closely resemble their fully random counterparts provided that $\delta / R \geq \sigma / R$.

The analysis of alternatively mistuned assemblies is greatly simplified by noting that such systems are in fact periodic arrangements of substructures each of which consists of two of the original subcomponents. For example, a chain of single-degreeof-freedom systems, such as shown in Fig. I.1, that is alternatively mistuned, i.e. with $\delta k_{j}=(-1)^{j} \beta$, represents a periodic assembly of two-degree-offreedom models having stiffnesses $k_{t}-\beta$ and $k_{t}+\beta$, respectively. In this light, the corresponding equations of motion, Eq. (I.1), can be rewritten in the form

$$
\begin{aligned}
& \left(\begin{array}{cc}
m & 0 \\
0 & m
\end{array}\right)\left(\begin{array}{c}
\ddot{X}_{2 j} \\
\ddot{X}_{2 j+1}
\end{array}\right)+\left(\begin{array}{cc}
c & 0 \\
0 & c
\end{array}\right)\left(\begin{array}{c}
\dot{X}_{2 j} \\
\dot{X}_{2 j+1}
\end{array}\right) \\
& +\left(\begin{array}{cc}
k_{t}+2 k_{\mathrm{C}}+\beta & -k_{\mathrm{C}} \\
-k_{\mathrm{C}} & k_{t}+2 k_{\mathrm{C}}-\beta
\end{array}\right)\left(\begin{array}{c}
X_{2 j} \\
X_{2 j+1}
\end{array}\right) \\
& -\left(\begin{array}{cc}
0 & k_{\mathrm{C}} \\
0 & 0
\end{array}\right)\left(\begin{array}{c}
X_{2 j-2} \\
X_{2 j-1}
\end{array}\right)-\left(\begin{array}{cc}
0 & 0 \\
k_{\mathrm{C}} & 0
\end{array}\right)\left(\begin{array}{c}
X_{2 j+2} \\
X_{2 j+1}
\end{array}\right) \\
& =\left(\begin{array}{c}
F_{2 j}(t) \\
F_{2 j+1}(t)
\end{array}\right) .
\end{aligned}
$$

Focusing on the determination of the localization factor of this system, consider the simple excitation

$$
F_{j}(t)= \begin{cases}F_{0} \mathrm{e}^{\mathrm{i} \omega t} & j=0, \\ 0 & j \neq 0,\end{cases}
$$

and denote the corresponding response as

$$
\underline{Y}_{j}(t)=\left(\begin{array}{c}
X_{2 j}(t) \\
X_{2 j+1}(t)
\end{array}\right)=\left(\begin{array}{c}
X_{2 j}^{\prime} \\
X_{2 j+1}^{\prime}
\end{array}\right) \mathrm{e}^{\mathrm{i} \omega t}=\underline{Y}_{j}^{\prime} \mathrm{e}^{\mathrm{i} \omega t} .
$$

Combining Eqs. (13)-(15), it is found that

$$
\begin{gathered}
\left(\begin{array}{cc}
k_{t}+2 k_{\mathrm{C}}+\beta-m \omega^{2}+\mathrm{i} \omega c & -k_{\mathrm{C}} \\
-k_{\mathrm{C}} & k_{t}+2 k_{\mathrm{C}}-\beta-m \omega^{2}+\mathrm{i} \omega c
\end{array}\right) \underline{Y}_{j}^{\prime} \\
-\left(\begin{array}{cc}
0 & k_{\mathrm{C}} \\
0 & 0
\end{array}\right) \underline{Y}_{j-1}^{\prime}-\left(\begin{array}{ll}
0 & 0 \\
k_{\mathrm{C}} & 0
\end{array}\right) \underline{Y}_{j+1}^{\prime}=\underline{0}
\end{gathered}
$$

for $j>0$. The above difference equation has constant coefficients so that its solution can be expressed in the form $\underline{Y}_{j}^{\prime}=\underline{Y}_{0}^{\prime} \rho^{j}$ for some complex number $\rho$. Introducing this expression in Eq. (16) yields the characteristic equation

$$
\rho^{2}+\left[2-\frac{\Delta^{2}-\beta^{2}}{k_{\mathrm{C}}^{2}}\right] \rho+1=0,
$$

where $\Delta=k_{t}+2 k_{\mathrm{C}}-m \omega^{2}+\mathrm{i} \omega c=k_{\mathrm{C}}[2-\alpha+\mathrm{i} \delta / R]$. Of the two roots of the above quadratic equation, which are inverse of each other, the one with a magnitude larger than one must be excluded from the solution since it corresponds to an unbounded growth of the vector $\underline{Y}_{j}^{\prime}$ as $j \rightarrow \infty$. Thus, the response of the chain of single-degree-of-freedom systems is exactly of the form $\underline{Y}_{j}^{\prime}=\underline{Y}_{0}^{\prime} \rho^{j}$ with

$$
\begin{gathered}
\rho=\rho(\beta)=-\left[1-\frac{\Delta^{2}-\beta^{2}}{2 k_{\mathrm{C}}^{2}}\right] \\
\pm \sqrt{\left[1-\frac{\Delta^{2}-\beta^{2}}{2 k_{\mathrm{C}}^{2}}\right]^{2}-1}
\end{gathered}
$$

with the sign in front of the square root such that $|\rho| \leq 1$. Then, the localization factor $\gamma(\beta)$ corresponding to fluctuations in stiffnesses $\pm \beta$ is obtained from Eq. (9) as

$$
\gamma(\beta)=-\frac{1}{2} \ln |\rho(\beta)| .
$$

In a fully random mistuned system, the amplitudes of the short wavelength components of the local modeling of the fluctuations in structural properties vary spatially. Thus, to account for these changes, it is necessary to model the mistuning strength $\beta$ as a random variable with standard deviation $\sigma$ in Eqs. (18) and (19). Then, the localization factor is

$$
\gamma=E[\gamma(\beta)]=\int_{-\infty}^{\infty} \gamma(\beta) p_{\delta k}(\beta) \mathrm{d} \beta,
$$

where $p_{\delta k}(\beta)$ is the probability density function of the stiffness fluctuations $\delta k_{j}$. Assuming a uniform 
distribution, as Castanier and Pierre (1993), yields

$$
\gamma=\frac{-1}{4 \sigma \sqrt{3}} \int_{-\sigma \sqrt{3}}^{\sigma \sqrt{3}} \ln |\rho(\beta)| \mathrm{d} \beta
$$

To assess the validity of the present alternate mistuning model, the above approximation of the localization factor was compared to the exact values obtained by Castanier and Pierre (1993) for the situation $\alpha=2$ (midband frequency, $\Delta=\mathrm{i} k_{\mathrm{C}} \delta / R$ ) and $\delta / R=\sigma / R$ (see Fig. 5). The excellent matching seen in this figure in the mild to strong localization range, i.e. $\delta / R=\sigma / R \geq 1$, clearly demonstrates the worth of the present model not only in the strong localization limit but also and more importantly in the weak to strong transition. In fact, the use of the weak localization limit, Eq. (10), for $\delta / R=\sigma /$ $R \leq 1$ and the alternate mistuning result, Eq. (21), for $\delta / R=\sigma / R \geq 1$ provides an excellent approximation of the localization factor for all values of $\delta / R=\sigma / R$.

\section{Distribution of the Forced Response, $\delta>\sigma$}

The excellent results shown in Fig. 5 demonstrate that alternatively and randomly mistuned systems are similarly localized when $\delta / R \geq 1$ and $\delta / R \geq \sigma / R$. Thus, it might be expected that the forced responses of these two different systems would also display similar characteristics. To verify this expectation, consider the $r$ th engine order excitation specified by Eq. (I.2) and let the corresponding response of the alternatively mistuned disk be

$$
\underline{Y}_{j}(t)=\left(\begin{array}{c}
X_{2 j}(t) \\
X_{2 j+1}(t)
\end{array}\right)=\left(\begin{array}{c}
\tilde{X}_{2 j} \mathrm{e}^{\mathrm{i}[\omega t+(2 j-1) \Delta \psi]} \\
\tilde{X}_{2 j+1} \mathrm{e}^{\mathrm{i}[\omega t+2 j \Delta \psi]}
\end{array}\right)
$$

Introducing Eqs. (I.2) and (22) in Eq. (13) yields the set of equations

$$
\begin{aligned}
& -k_{\mathrm{C}} \mathrm{e}^{\mathrm{i} \Delta \psi} \tilde{X}_{2 j+1}-k_{\mathrm{C}} \mathrm{e}^{-\mathrm{i} \Delta \psi} \tilde{X}_{2 j-1} \\
& +\left(k_{t}-m \omega^{2}+\beta+2 k_{\mathrm{C}}+\mathrm{i} \omega c\right) \tilde{X}_{2 j}=\tilde{F}_{0}
\end{aligned}
$$

and

$$
\begin{aligned}
& -k_{\mathrm{C}} \mathrm{e}^{\mathrm{i} \Delta \psi} \tilde{X}_{2 j+2}-k_{\mathrm{C}} \mathrm{e}^{-\mathrm{i} \Delta \psi} \tilde{X}_{2 j} \\
& \quad+\left(k_{t}-m \omega^{2}-\beta+2 k_{\mathrm{C}}+\mathrm{i} \omega c\right) \tilde{X}_{2 j+1}=\tilde{F}_{0}
\end{aligned}
$$

Further, the equivalence between this mistuned bladed disk and a tuned assembly of two-degreeof-freedom systems suggests the existence of a solution of the form $\tilde{X}_{2 j}=$ const $=\tilde{X}_{\mathrm{e}}$ and $\tilde{X}_{2 j+1}=$ const $=\tilde{X}_{\mathrm{o}}$ for all $j$. With this assumption, Eqs. (23) and (24) reduce to two linear algebraic equations, the solution of which is

$$
\tilde{X}_{\mathrm{e}}=\tilde{F}_{0} \frac{\Delta-\beta+2 k_{\mathrm{C}} \cos \Delta \psi}{\Delta^{2}-\beta^{2}-4 k_{\mathrm{C}}^{2} \cos ^{2} \Delta \psi}
$$

and

$$
\tilde{X}_{\mathrm{o}}=\tilde{F}_{0} \frac{\Delta+\beta+2 k_{\mathrm{C}} \cos \Delta \psi}{\Delta^{2}-\beta^{2}-4 k_{\mathrm{C}}^{2} \cos ^{2} \Delta \psi}
$$

At resonance, the frequency $\omega$ is given by Eq. (I.3) and $\Delta=2 k_{\mathrm{C}} \cos \Delta \psi+\mathrm{i} \omega c$ so that

$$
\begin{aligned}
\tilde{X}_{\mathrm{o}, \mathrm{e}} & =\tilde{F}_{0} \frac{4 k_{\mathrm{C}} \cos \Delta \psi+\mathrm{i} \omega c \pm \beta}{-\left(\omega^{2} c^{2}+\beta^{2}\right)+4 i \omega c k_{\mathrm{C}} \cos \Delta \psi} \\
& =U_{\mathrm{o}, \mathrm{e}}+\mathrm{i} V_{\mathrm{o}, \mathrm{e}}
\end{aligned}
$$

The validity of this model of the forced response of mistuned bladed disks will be assessed by comparing the behavior of the expected (mean) value of the real part of the response, i.e. $E\left[U_{\mathrm{o}, \mathrm{e}}\right]$, computed from the above expression and from thorough Monte Carlo simulations of randomly mistuned disks. The selection of $E\left[U_{\mathrm{o}, \mathrm{e}}\right]$ for the testing of Eq. (27) is motivated by the high sensitivity of this quantity with respect to the coupling stiffness in the weak to strong localization transition region, as demonstrated by Mignolet and $\mathrm{Hu}$ (1998).

Taking the real part of $\tilde{X}_{\mathrm{o}, \mathrm{e}}$ yields

$$
U_{\mathrm{o}, \mathrm{e}}=\tilde{F}_{0} \frac{-4 k_{\mathrm{C}} \cos \Delta \psi \beta^{2} \mp \beta\left(\omega^{2} c^{2}+\beta^{2}\right)}{\left(\omega^{2} c^{2}+\beta^{2}\right)^{2}+16 \omega^{2} c^{2} k_{\mathrm{C}}^{2} \cos ^{2} \Delta \psi}
$$


so that

$$
\begin{aligned}
E\left[U_{\mathrm{o}, \mathrm{e}}\right]= & -4 k_{\mathrm{C}} \cos \Delta \psi \tilde{F}_{0} \\
& \times E\left[\frac{\beta^{2}}{\left(\omega^{2} c^{2}+\beta^{2}\right)^{2}+16 \omega^{2} c^{2} k_{\mathrm{C}}^{2} \cos ^{2} \Delta \psi}\right]
\end{aligned}
$$

if the stiffness fluctuations $\delta k_{j}$ are symmetric. In the specific case of a zero-mean Gaussian distribution of the random variables $\delta k_{j}$ and $\beta$, it is found that (Abramowitz and Stegun, 1972)

$$
E\left[U_{\mathrm{o}, \mathrm{e}}\right]=-\left(\frac{\tilde{F}_{0}}{c \omega}\right) \sqrt{\pi} \operatorname{Im}\left[z \mathrm{e}^{z^{2}} \operatorname{erfc}(z)\right]
$$

where $z=\left(c^{2} \omega^{2}+\mathrm{i} 4 k_{\mathrm{C}} c \omega \cos \Delta \psi\right) /\left(2 \sigma^{2}\right)$. Further, $\operatorname{Im}(w)$ and $\operatorname{erfc}(w)$ denote the imaginary part and the complimentary error function of a complex number $w$ (Abramowitz and Stegun, 1972). To test the accuracy of Eq. (30) in predicting the sensitivity of the mean value $E\left[U_{\mathrm{o}, \mathrm{e}}\right]$ with respect to the coupling stiffness $k_{\mathrm{C}}$, Monte Carlo simulations were conducted with a third engine order excitation and the damping coefficient $c=1.443 \mathrm{Ns} / \mathrm{m}$ for different values of $k_{\mathrm{C}}$. The comparison of these results with their counterparts computed from Eq. (30) with $\sigma^{2}=E\left[\beta^{2}\right]=E\left[\delta k_{j}^{2}\right]$ first demonstrated that the latter values were typically three times larger than the former ones. This lack of quantitative matching of the response is not unexpected since the $s=N / 2$ harmonic of the mistuning pattern is not its only component that affects the response of the system, it only is the one yielding the largest effect.

Although the quantitative prediction of the effects of mistuning is desired, recent efforts in this area (e.g. Mignolet and Lin, 1996; Mignolet and $\mathrm{Hu}, 1998$ ) have shown that the development of a qualitative model displaying the correct physical characteristics of the response represents a critical step in this derivation. In this light, it is important to assess if Eq. (30) accurately matches the physical features, such as high sensitivity with respect to the coupling stiffness, location of the maximum, etc., of the mean of the real part of the response. To this end, both the exact values of this quantity and their approximations from Eq. (30) were normalized by their respective maxima. The excellent matching between these two sets of results in the domain $k_{\mathrm{C}} \in[0,20,000] \mathrm{N} / \mathrm{m}$ (see Fig. 6) demonstrates that indeed Eq. (30) can reliably be used to uncover the physical characteristics of the average response of weakly and mildly coupled bladed disks. Note finally that a continued fraction expansion of the error function exists (see Abramowitz and Stegun 1972) that leads to the accurate and simple approximation of Eq. (30) in the form

$$
E\left[U_{\mathrm{o}, \mathrm{e}}\right] \approx \frac{-4 k_{\mathrm{C}} \cos \Delta \psi \sigma^{2}}{\left(\omega^{2} c^{2}+3 \sigma^{2}\right)^{2}+16 \omega^{2} c^{2} k_{\mathrm{C}}^{2} \cos ^{2} \Delta \psi} \tilde{F}_{0}
$$

\section{Distribution of the Maximum Response}

The determination of the distribution of the maximum amplitude of response observed on a typical disk is an especially important problem in the present context since this probability density function is associated with the fatigue life of the disk. Unfortunately, probability theory provides only a limited number of results pertinent to the characteristics of the maximum $A_{\max }$ of a series of random variables $A_{j}, j=1,2, \ldots, N$. In particular, if these random variables are independent it can be shown that

$$
F_{A_{\max }}(a)=\prod_{j=1}^{N} F_{A_{j}}(a)
$$

where $F_{A_{j}}(a)$ is the cumulative distribution function of $A_{j}$ and is defined in terms of its probability density function, $p_{A_{j}}(a)$, as

$$
F_{A_{j}}(a)=\int_{-\infty}^{a} p_{A_{j}}(a) \mathrm{d} a
$$

When the number $N$ of random variables becomes large, it can be shown (Gumbel, 1958) 


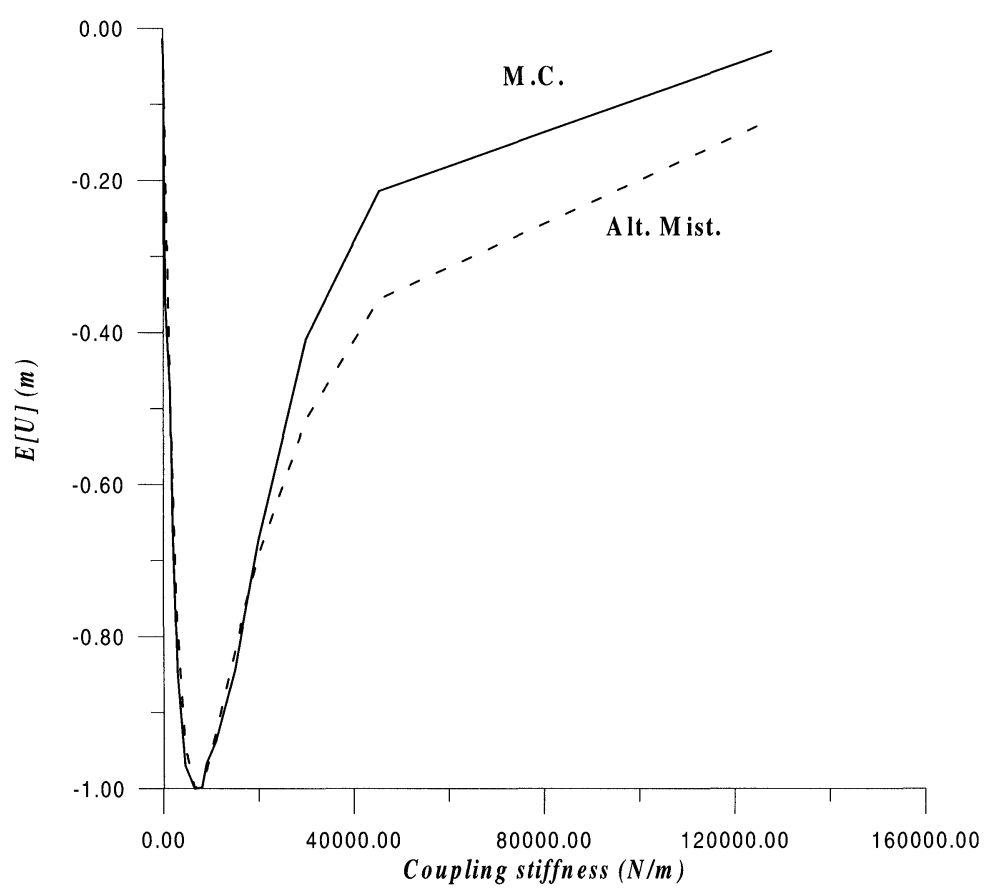

FIGURE 6 Mean value of the real part of the response as a function of the coupling stiffness obtained from Monte Carlo simulation ("M.C.") on a fully random disk and from the alternate mistuning model ("Alt. Mist.").

that the cumulative distribution function of the maximum corresponding to Eq. (32) admits, under mild conditions, one of several limit forms. In particular, when an upper bound of the variables $A_{j}$ exists, as can be justified in the present context by relying on the work of Whitehead (1966), it has been shown that

$$
F_{A_{\max }}(a) \rightarrow \exp \left[-\alpha\left(a_{0}-a\right)^{\beta}\right] \quad \text { as } N \rightarrow \infty
$$

for some parameters $\alpha, \beta$ and $a_{0}$. Although Eq. (32) is valid only for independent random variables $A_{j}$, it has been shown (Gumbel, 1958) that Eq. (34) is also applicable if these variables are correlated only by small groups. This last result is particularly interesting in the present context since the amplitudes of response of neighborhooding blades are independent of each other only when $k_{\mathrm{C}}=0$ so that Eq. (32) can be shown to hold only in extremely weakly coupled disks. However, the assumption of correlation by small groups is valid in the entire range of weak coupling since localization prevents the flow of "information", or equivalently, leads only to very small correlations between the responses of blades that are far apart. The analysis of partially mistuned bladed disks provides in particular a basis for formally assessing the existence and magnitude of correlations. For example, the modified 1-blade mistuning model leads to a nonzero correlation between the responses of a blade and of its two closest neighbors on either side only.

With these comments, it could be expected that the Weibull-type distribution, Eq. (34), would accurately model the maximum amplitude of response for small coupling stiffnesses. To demonstrate this fact, extensive Monte Carlo simulations were conducted from which the probability density function and the first three moments of the maximum amplitude of response on the disk were obtained. Then, the parameters $\alpha, \beta$ and $a_{0}$ of the Weibulltype distribution, Eq. (34), were determined by 
matching the first three moments estimated by simulation to their model counterparts, i.e.

$$
E\left[\left(a_{0}-a\right)^{k}\right]=\alpha^{-k / \beta} \Gamma\left(1+\frac{k}{\beta}\right),
$$

where the symbol $\Gamma(\cdot)$ denotes the gamma function. Then, the appropriateness of the Weibull-type model was assessed by comparing the corresponding probability density function

$$
p_{A_{\max }}(a)=\alpha \beta\left(a_{0}-a\right)^{\beta-1} \exp \left[-\alpha\left(a_{0}-a\right)^{\beta}\right]
$$

to its Monte Carlo counterpart. The excellent matching between these two sets of curves for $k_{\mathrm{C}}=$ 2,000 N/m (see Fig. 7) clearly demonstrates the reliability of the Weibull-type model, Eqs. (34)(36), in representing the distribution of the maximum amplitude of response of weakly coupled mistuned bladed disks. In fact, it can be seen from Figs. 8 and 9 that, although the correlation arguments developed above are only valid for small coupling stiffnesses, the Weibull-type probability density function provides nevertheless an excellent model of the characteristics of the maximum amplitude for all coupling levels!

This important result indicates that the knowledge of the first three moments of this random variable provides a complete characterization of its properties, including the parameter $a_{0}$, which represents the largest amplitude of response that a blade can experience. This observation can lead to substantial computational savings since the accurate estimation of the coefficient $a_{0}$ by the Monte Carlo method is a much more demanding task than the determination of the moments as it is associated with rare events and thus requires a very large number of simulations.

\section{Simulation}

The previous sections have emphasized the consideration of harmonically and partially mistuned bladed disks for the derivation of analytical results

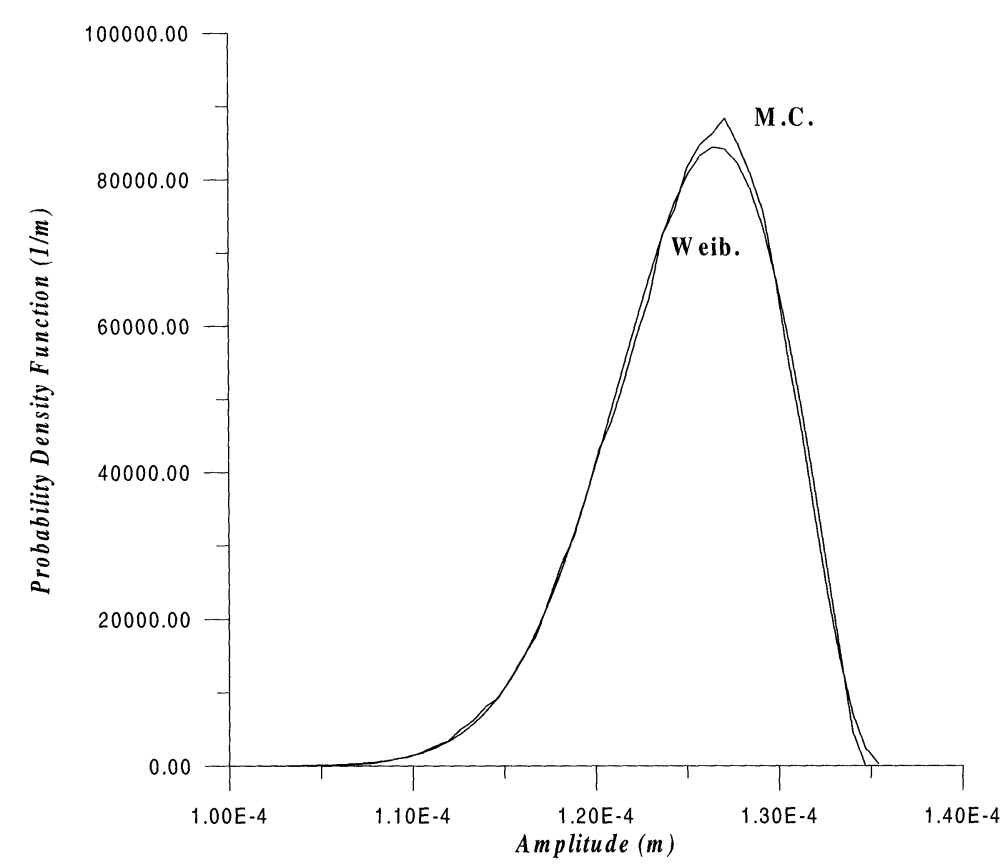

FIGURE 7 Probability density function of the maximum response obtained by Monte Carlo simulation ("M.C.") and its Weibull distribution approximation ("Weib."), $k_{\mathrm{C}}=2,000 \mathrm{~N} / \mathrm{m}$. 


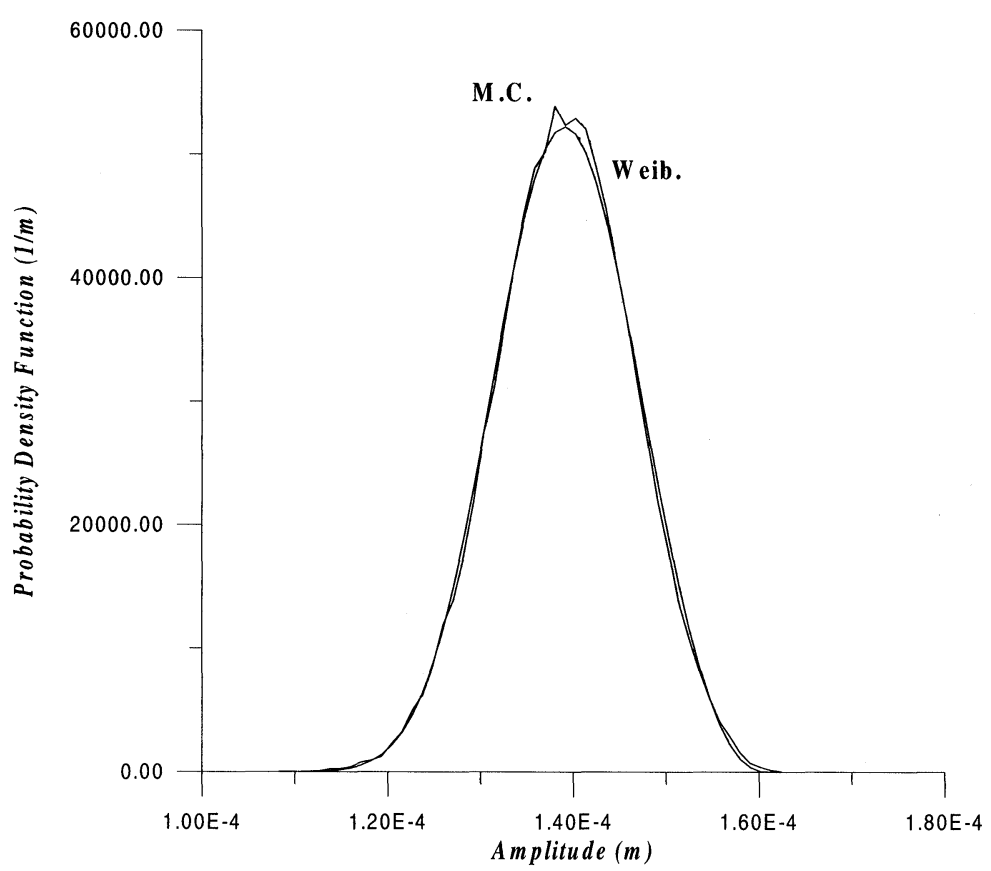

FIGURE 8 Probability density function of the maximum response obtained by Monte Carlo simulation ("M.C.") and its Weibull distribution approximation ("Weib."), $k_{\mathrm{C}}=6,500 \mathrm{~N} / \mathrm{m}$.

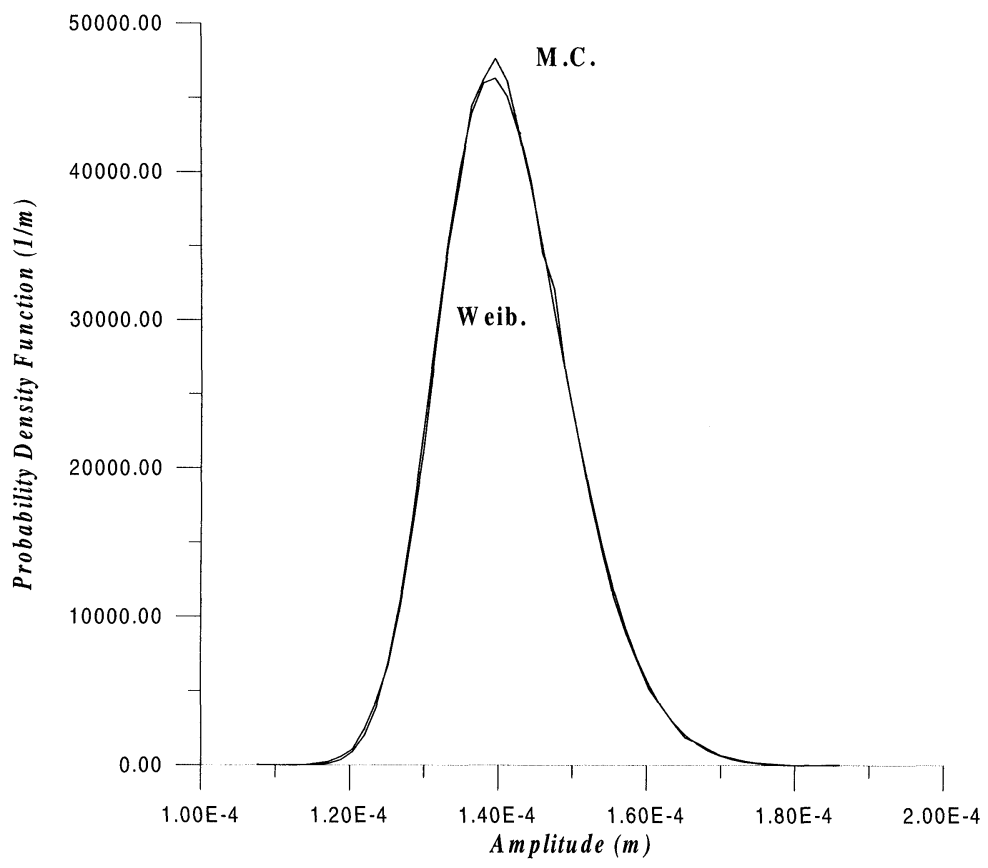

FIGURE 9 Probability density function of the maximum response obtained by Monte Carlo simulation ("M.C.") and its Weibull distribution approximation ("Weib."), $k_{\mathrm{C}}=20,000 \mathrm{~N} / \mathrm{m}$. 
and models that are applicable to the general case of fully random structural properties. It should however be restated that these systems can also serve as bases for the development of reliable and computationally efficient simulation schemes. This potential application was clearly demonstrated in the case of partially mistuned disks (see Figs. 1-3) but it also holds in connection with harmonic mistuning. Specifically, in the case of strongly coupled systems, the response of the blades can be expressed in the form of Eq. (I.28) with two terms, i.e.

$$
\tilde{X}_{j}=\tilde{X}_{j}^{(0)}+\tilde{X}_{j}^{(1)},
$$

where the components $\tilde{X}_{j}^{(0)}$ and $\tilde{X}_{j}^{(1)}$ can be efficiently computed from Eqs. (I.29)-(I.34).

\section{SUMMARY}

In this second paper, the exact characteristics of partially mistuned bladed disks have been investigated to provide phenomenological insights into and analytical results on the effects of fully random mistuning on the response of these systems. The contributions of the present investigation can be summarized as follows.

(1) Both the qualitative and quantitative aspects of the forced response of weakly coupled bladed disks can accurately be predicted by considering partially mistuned system in which only a few, from 3 to 9 , consecutive blades have structural properties that differ from their design counterparts. This finding was then shown to produce dramatic computational savings in the computation of the random forced response of mistuned weakly coupled bladed disks.

(2) The findings of Part I were used to demonstrate that the response of weakly coupled bladed disks with alternate mistuning exhibits many characteristics that not only qualitatively but also quantitatively match their counterparts for a system with random variations in structural properties provided that the damping ratio is larger than or equal to half of the coefficient of variation of the fluctuations in stiffnesses. As simple examples of the wide range applicability of this property, closed form approximations of the localization factor and of the mean of the forced response were derived that are very reliable in the entire domain of mild to very weak coupling.

(3) The presence of only short range correlations between the response of different blades that is characteristic of both randomly and partially mistuned weakly coupled disks indicated that the maximum amplitude of response of such systems follows a Weibull-type distribution. Extensive comparisons with Monte Carlo simulations demonstrated that the Weibull-type model is in fact accurate in the entire range of blade-to-blade coupling, from very weak to very strong! This observation was shown to yield a substantial reduction in the number of simulations that would be required to obtain a reliable approximation of the distribution of the maximum response and of its most important features.

\section{Acknowledgments}

The financial support of this work by the Naval Surface Warfare Center, Caderock Division, Department of the Navy, Bethesda, MD 20084, is gratefully acknowledged. Further, the first author (M.P.M.) wishes to express his deep gratitude to Prof. J.C.P. Mignolet for his continued support of this and other research efforts.

\section{References}

Abramowitz, M. and Stegun, I.A., 1972. Handbook of Mathematical Functions, Dover.

Castanier, M.P. and Pierre, C., 1993. Individual and interactive mechanisms for localization and dissipation in a monocoupled nearly-periodic structure, Journal of Sound and Vibration, 168, 479-505.

Gumbel, E.J., 1958. Statistics of Extremes, Columbia University Press. 
Mignolet, M.P. and Hu, W., 1998. Direct prediction of the effects of mistuning on the forced response of bladed disks, Journal of Engineering for Gas Turbines and Power, 120, 626-634.

Mignolet, M.P. and Lin, C.C., 1996. A novel limit distribution for the analysis of randomly mistuned bladed disks, ASME Paper 96-GT-414, Presented at the 41th International Gas Turbine and Aeroengine Congress and Exposition, United Kingdom.
Wei, S.-T. and Pierre, C., 1988. Localization phenomena in mistuned assemblies with cyclic symmetry - Part II: Forced vibrations, Journal of Vibration, Acoustics, Stress, and Reliability in Design, 110, 439-449.

Whitehead, D.S., 1966. Effect of mistuning on the vibration of turbomachines blades induced by wakes, Journal of Mechanical Engineering Science, 8, 15-21. 


\section{ait \\ ENERGY MATERIALS}

M A N E Y publishing

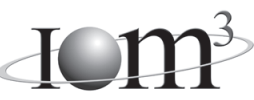

\section{Materials Science \& Engineering for Energy Systems}

Maney Publishing on behalf of the Institute of Materials, Minerals and Mining

The Institute of Materials, Minerals \& Mining

Economic and environmental factors are creating ever greater pressures for the efficient generation, transmission and use of energy. Materials developments are crucial to progress in all these areas: to innovation in design; to extending lifetime and maintenance intervals; and to successful operation in more demanding environments. Drawing together the broad community with interests in these areas, Energy Materials addresses materials needs in future energy generation, transmission, utilisation, conservation and storage. The journal covers thermal generation and gas turbines; renewable power (wind, wave, tidal, hydro, solar and geothermal); fuel cells (low and high temperature); materials issues relevant to biomass and biotechnology; nuclear power generation (fission and fusion); hydrogen generation and storage in the context of the 'hydrogen economy'; and the transmission and storage of the energy produced.

As well as publishing high-quality peer-reviewed research, Energy Materials promotes discussion of issues common to all sectors, through commissioned reviews and commentaries. The journal includes coverage of energy economics and policy, and broader social issues, since the political and legislative context influence research and investment decisions.

\section{CALL FOR PAPERS}

Contributions to the journal should be submitted online at http://ema.edmgr.com

To view the Notes for Contributors please visit: www.maney.co.uk/journals/notes/ema

Upon publication in 2006, this journal will be available via the Ingenta Connect journals service. To view free sample content online visit: www.ingentaconnect.com/content/maney

For further information please contact:

Maney Publishing UK

Tel: +44 (0)113 2497481 Fax: +44 (0)1132486983 Email: subscriptions@maney.co.uk

or

Maney Publishing North America

Tel (toll free): 8662975154 Fax: 6173546875 Email: maney@maneyusa.com

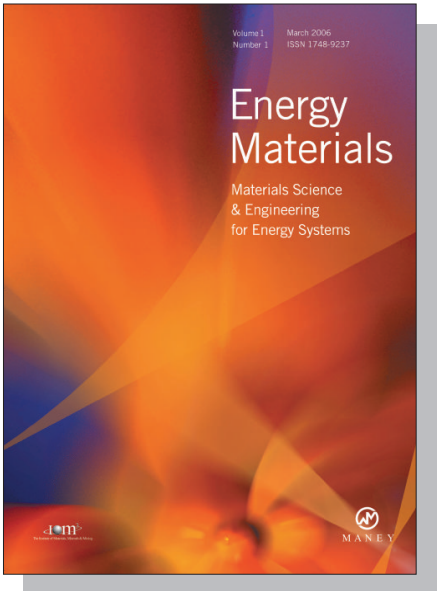

EDITORS

Dr Fujio Abe

NIMS, Japan

Dr John Hald, IPL-MPT, Technical University of Denmark, Denmark

Dr R Viswanathan, EPRI, USA

\section{SUBSCRIPTION INFORMATION}

Volume 1 (2006), 4 issues per year

Print ISSN: 1748-9237 Online ISSN: 1748-9245

Individual rate: $£ 76.00 / U S \$ 141.00$

Institutional rate: $£ 235.00 /$ US $\$ 435.00$

Online-only institutional rate: $£ 199.00 / U S \$ 367.00$

For special $\mathrm{IOM}^{3}$ member rates please email

subscriptions@maney.co.uk

\section{For further information or to subscribe online please visit www.maney.co.uk}



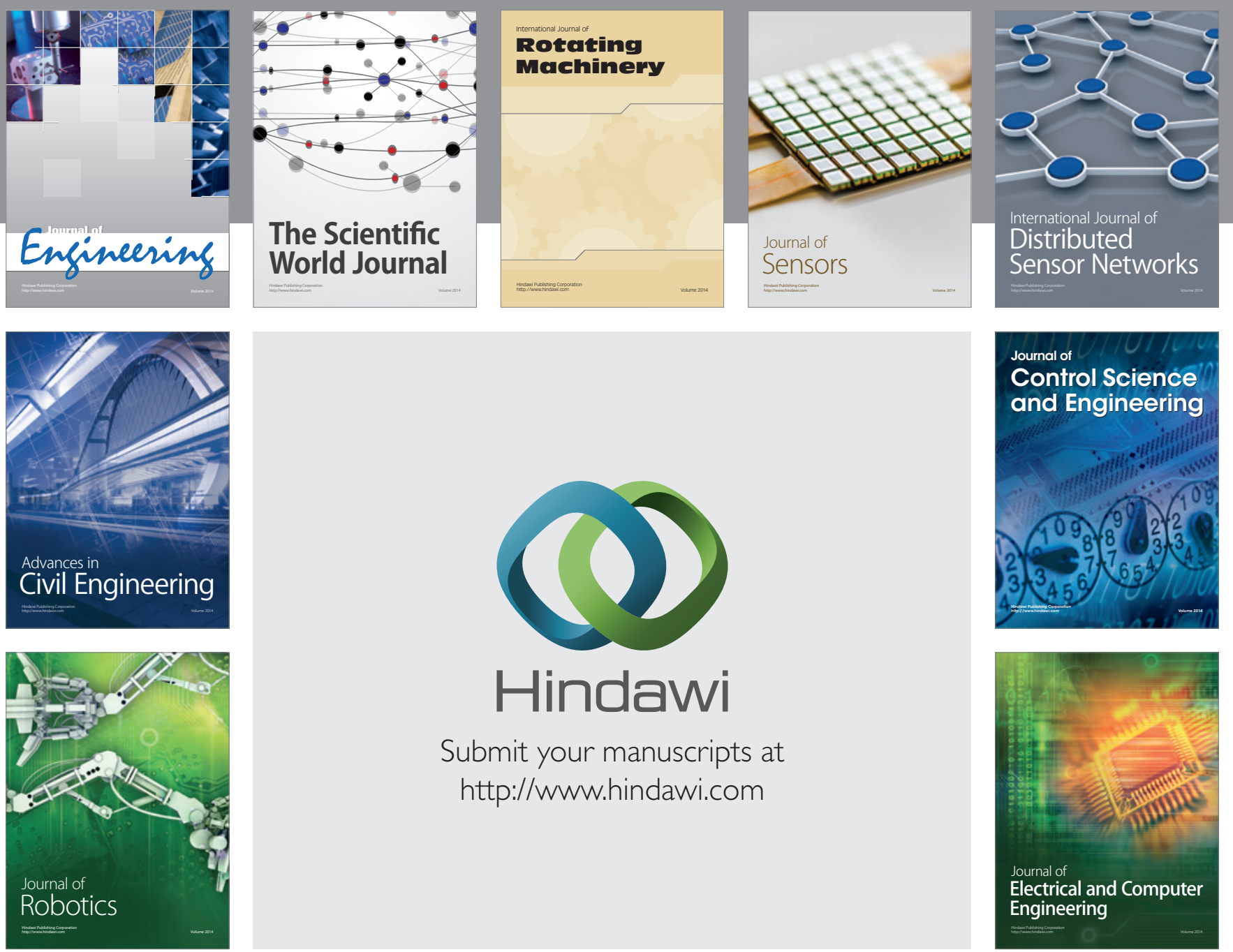

Submit your manuscripts at

http://www.hindawi.com
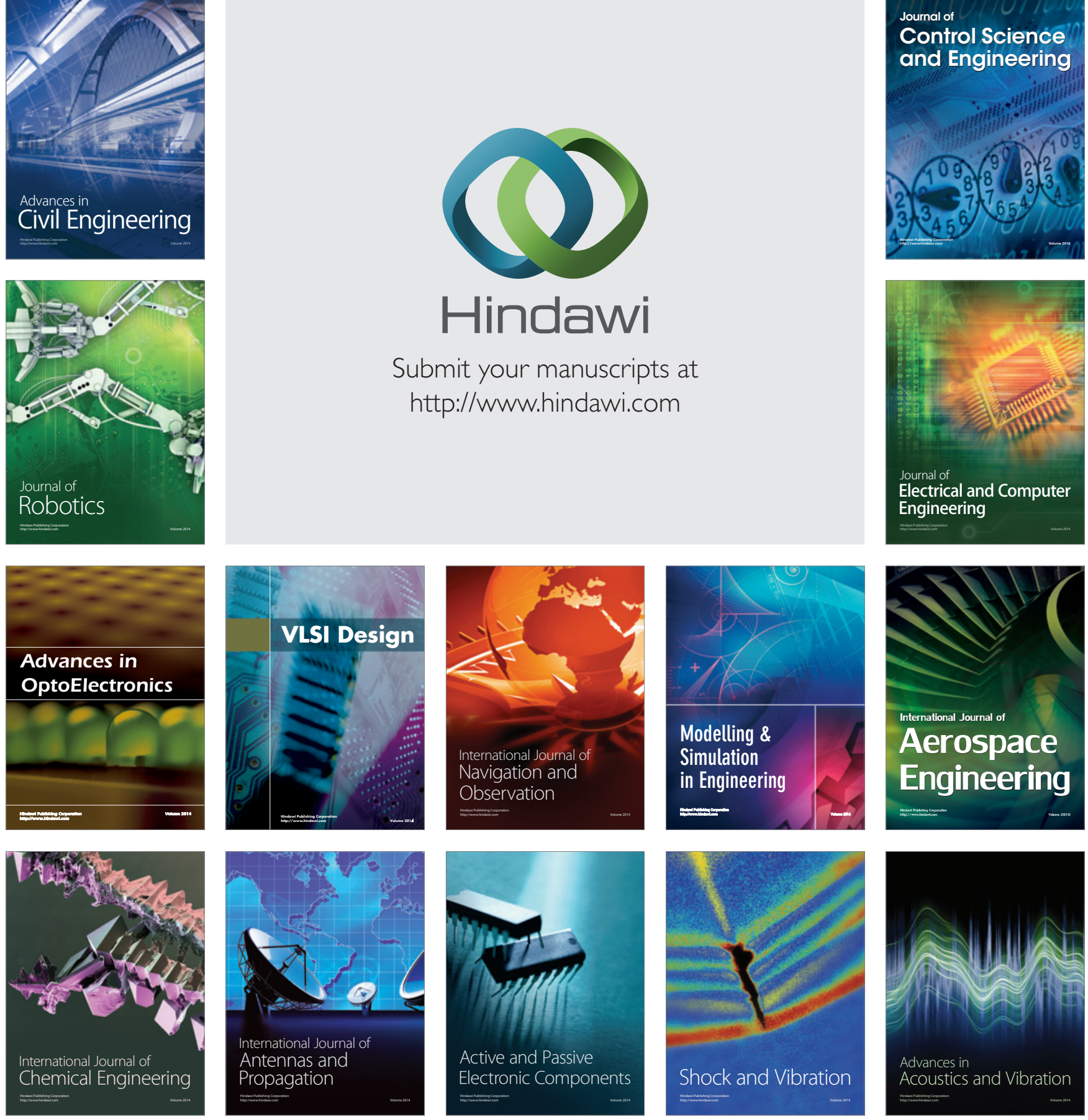